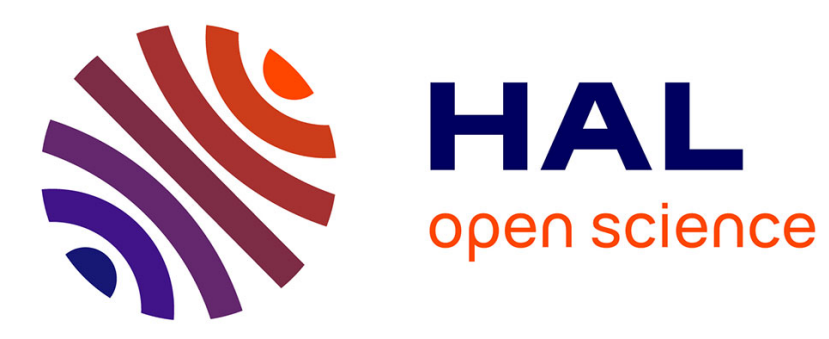

\title{
Total Internal Reflection Tip-Enhanced Raman Spectroscopy of Cytochrome C
}

David Talaga, Andrew Bremner, Thierry Buffeteau, Renaud Vallée, Sophie Lecomte, Sébastien Bonhommeau

\section{- To cite this version:}

David Talaga, Andrew Bremner, Thierry Buffeteau, Renaud Vallée, Sophie Lecomte, et al.. Total Internal Reflection Tip-Enhanced Raman Spectroscopy of Cytochrome C. Journal of Physical Chemistry Letters, 2020, 11, pp.3835-3840. hal-02899317

\section{HAL Id: hal-02899317 https://hal.science/hal-02899317}

Submitted on 15 Jul 2020

HAL is a multi-disciplinary open access archive for the deposit and dissemination of scientific research documents, whether they are published or not. The documents may come from teaching and research institutions in France or abroad, or from public or private research centers.
L'archive ouverte pluridisciplinaire HAL, est destinée au dépôt et à la diffusion de documents scientifiques de niveau recherche, publiés ou non, émanant des établissements d'enseignement et de recherche français ou étrangers, des laboratoires publics ou privés. 


\section{Total Internal Reflection Tip-Enhanced Raman}

\section{Spectroscopy of Cytochrome C}

David Talaga,,$^{\dagger}$ Andrew Bremner, ${ }^{\dagger \$}$ Thierry Buffeteau, ${ }^{\dagger}$ Renaud A.L. Vallée, ${ }^{,}$Sophie Lecomte, ${ }^{\S}$ and Sébastien Bonhommeau ${ }^{*}+\dot{T}$

${ }^{\dagger}$ Univ. Bordeaux, CNRS, Bordeaux INP, ISM, UMR 5255, F-33400 Talence, France

${ }^{\$}$ Colorado School of Mines, Golden, CO 80401 USA

${ }^{*}$ Univ. Bordeaux, CNRS, CRPP, UMR 5031, F-33600 Pessac, France

${ }^{\S}$ Univ. Bordeaux, CNRS, CBMN, UMR 5248, F-33600 Pessac, France

* To whom correspondence should be addressed. E-mail: sebastien.bonhommeau@u-bordeaux.fr

ABSTRACT. Surface and tip-enhanced Raman spectroscopies in total internal reflection (TIRSERS and TIR-TERS) are used to characterize the oxidation, spin and ligation state of cytochrome $\mathrm{C}(\mathrm{Cc})$ molecules electrostatically bound on hydrophilic thiol self-assembled monolayer. TIR-SERS spectra of this model hemoprotein show marker bands typical of the 6cLS ferric state of Cc. The performances of the TIR-TERS technique as a function of the incidence angle are described, showing in particular a significant electromagnetic enhancement of the Raman signal under $p$-polarized light excitation. TIR-TERS spectra of Cc confirm the 6cLS ferric state assignment deduced from TIR-SERS spectra, thus demonstrating the possibility to 
probe with nanoscale spatial resolution the $6 \mathrm{cLC}$ oxidized form of $\mathrm{Cc}$ that is potentially implicated in cell apoptotic processes. The minimal far-field contribution of the sample in TIRTERS also offers promising perspectives for future nanoscale chemical characterizations of photosensitive biomolecules in complex biological media.

\section{TOC GRAPHICS}

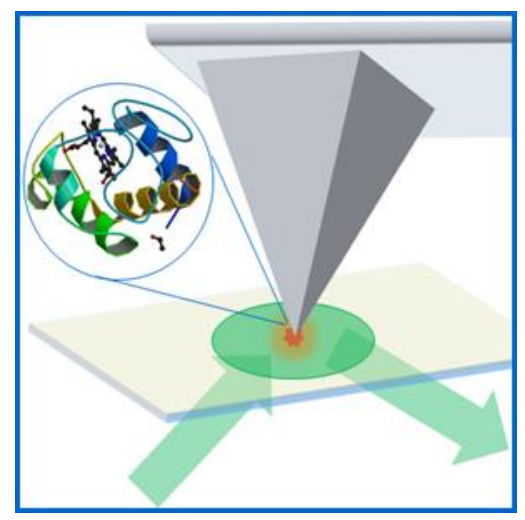

KEYWORDS Tip-enhanced Raman spectroscopy, Surface-enhanced Raman spectroscopy, Total internal reflection, Finite difference time domain, Cytochrome C.

TEXT. Tip-enhanced Raman spectroscopy (TERS) established itself as a powerful technique to characterize many biomolecules, such as nucleic acids, peptides/proteins and complex biological systems (cells, viruses, bacteria...). ${ }^{1}$ In TERS, a monochromatic propagative wave excites resonantly a tapered noble metal probe in order to generate an enhanced and confined electromagnetic field at the really end of this tip. The produced intense and localized evanescent field results from two enhancement mechanisms, namely the resonant plasmonic effect that is highly dependent on the material, the size, the shape and the surrounding medium of the TERS probe, and the non-resonant lighting-rod effect increasing the electron density at the tip apex. In 
addition, the resonant nanoantenna effect allows for the conversion of the evanescent (near) field into propagative (far) field, at the origin of the detected TERS signal. In traditional bottom, side or top-illumination configurations, ${ }^{1}$ a propagative-wave excitation lead to the irradiation of a tip portion with diffraction-limited dimensions much larger than the nanoscale apex size, which may induce the detection of parasitic signals generated by the tip sides. Furthermore, photosensitive molecules, such as hemoproteins, may be damaged when they are resonantly excited by direct light irradiation at the same time as the TERS tip, especially in bottom-illumination configuration. In order to limit these issues, an incident excitation in total internal reflection (TIR) geometry can be proposed. TIR-TERS has been recently applied to characterize a thin layer of copper phthalocyanine. ${ }^{2}$ Not only did the authors demonstrate the feasibility of TIRTERS measurements with nanoscale (better than $40 \mathrm{~nm}$ ) spatial resolution but they also revealed that the signal enhancement is more than 20 times over side-illumination configuration. However, in this case, TIR was realized through a prism instead of a high numerical aperture objective currently used in bottom-illumination TERS instruments that are often employed to probe biological specimens. ${ }^{1}$

Herein, we present a TIR-TERS technique implemented in a conventional bottom-illumination AFM-TERS instrument, by probing cytochrome $\mathrm{C}(\mathrm{Cc})$ under resonant excitations. $\mathrm{Cc}$ is a globular monoheme protein of relatively large size (ca. $3.4 \mathrm{~nm}$ in diameter) which acts as an electron carrier in the respiratory chain of aerobic organisms. ${ }^{3}$ It transfers electrons between the $\mathrm{Cc}$ reductase and $\mathrm{Cc}$ oxidase enzyme complexes which are both embedded in the mitochondrial membrane. It was shown to be implicated in the apoptotic processes via its transfer from the intermembrane space to the cytosol, one of the main enzymes acting in the reaction cascades of apoptosis. ${ }^{4,5}$ Apoptosis or programmed cell death is one of the mechanisms that may induce 
neuronal loss in the brain of persons suffering from Alzheimer's disease (AD). In the native form, $\mathrm{Cc}$ exists in ferric and ferrous states and shows a six-coordinated low-spin (6cLS) heme center. Only this native 6cLS state was suggested to characterize the form implicated in the apoptotic processes. ${ }^{6}$ In this state, the heme is axially bound to a methionine (Met) and an histidine (His) ligand. In non-native forms however, the axial Met ligand, that is the most labile ligand, is replaced or removed to form either six-coordinated high-spin (6cHS) or fivecoordinated high-spin (5cHS) species, respectively. ${ }^{6}$ These non-native forms were observed in both ferric and ferrous oxidation states. The oxidation, spin and ligation state of the Cc heme iron can be clearly identified by Raman and surface-enhanced Raman scattering (SERS) experiments under resonant excitation. ${ }^{6-14}$ These investigations showed that each $\mathrm{Cc}$ state was related to specific Raman bands in the $1300-1700 \mathrm{~cm}^{-1},{ }^{6,9-14}$ and may present an additional relevant feature at $\sim 750 \mathrm{~cm}^{-1} \cdot{ }^{10-12}$ SERS experiments on Cc molecules electrostatically bound to thiol selfassembled monolayers (SAMs) on $\mathrm{Ag}$ and $\mathrm{Au}$ electrodes (see Supporting Information for explanations about the interest of $\mathrm{Cc}$ attachment on alkanethiol SAMs) revealed that the 6cLS state of the adsorbed $\mathrm{Cc}$ prevails in the oxidized form while the 5cHS state seems to be predominant in the reduced form. ${ }^{7,8,10}$ All the observed bands were assigned to specific heme modes. However, amino acid bands were observed neither in RR nor in SERS. ${ }^{6,12}$ Only resonance tip-enhanced Raman spectroscopy (TERS) allowed vibration modes assigned to Histidine (His), Tyrosine (Tyr) and Phenylalanine (Phe) residues at 837, 932 and $1002 \mathrm{~cm}^{-1}$ respectively, ${ }^{12}$ and those characterizing the Cc oxidation and spin states, ${ }^{12-14}$ to be discerned. Nevertheless, very few TERS data on Cc molecules in the pure ferric 6cLS state were reported so far. ${ }^{14}$ 
Prior to the description of TIR-TERS, resonance TIR-SERS measurements on Cc molecules are presented to provide benchmark spectra for subsequent comparison with their TIR-TERS counterparts. The advantages of TIR-TERS are then emphasized in the light of the performances of TIR-Raman, using an experimental/theoretical approach. Finally, TIR-TERS is employed to probe Cc and confirm its oxidation, spin and ligation state.

Determination of the Cc state by TIR-SERS. TIR-SERS measurements of Cc molecules electrostatically bound to a SAM of 11-mercaptoundecanoic acid (MUA) covering a rough Ag film (see experimental details in Supporting Information) were carried out at 532nm irradiation wavelength. This allows for the resonant excitation of both $\mathrm{Cc}$ molecules and the localized surface plasmon resonance (LSPR) of the SERS-active Ag substrate. The UV-visible absorption spectrum of $\mathrm{Cc}$ in $0.2 \mathrm{mM}$ aqueous solution displays indeed a Soret band at $409 \mathrm{~nm}$, and a weaker Q band at 530nm, both originating from the porphyrin chromophore of the heme group (Figure 1A). Moreover, dark-field Rayleigh scattering microscopy (DFRSM) spectra of uncoated SERS substrates show LSPRs at $570 \mathrm{~nm}$ that are red shifted by $60 \mathrm{~nm}$ upon MUA coating (Figure 1B). The addition of $\mathrm{Cc}$ on the thiol SAM does not affect further LSPRs. Only the scattering intensity is progressively decreased upon increasing the thickness of the dielectric layer ( $\mathrm{Cc}$ and thiols) on top of the metal substrate (Figure 1B). For $532 \mathrm{~nm}$ excitation, the investigated $500-1800 \mathrm{~cm}^{-1}$ spectral range corresponds to the $546-588 \mathrm{~nm}$ region located near the maximum of the LSPR band of SERS substrates. This irradiation wavelength is thus perfectly suited to achieve resonance SERS measurements of Cc. In contrast, the excitation of the Soret band could lead to very intense resonance Raman signals of the heme center of $\mathrm{Cc}$, but the SERS enhancement of Raman signatures associated with both $\mathrm{Cc}$ and the underlying thiols would be quite weak, and inappropriate for the forthcoming comparison with TERS. 

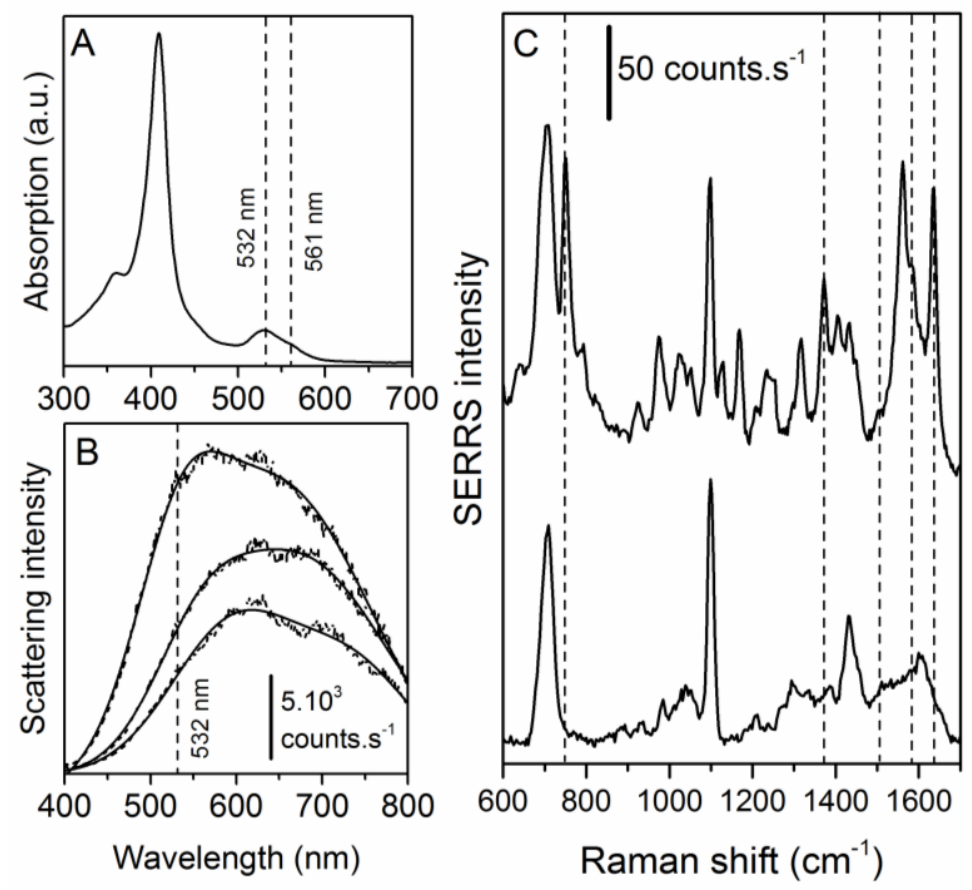

Figure 1. A) UV-visible absorption spectrum of an $0.2 \mathrm{mM}$ aqueous solution of Cc. Laser wavelengths used to excite the LSPR of the SERS substrates and the TERS tip are indicated. B) From top to bottom, DFRSM spectra of uncoated and MUA-coated silver SERS substrates as well as DFRSM spectrum of silver SERS substrate coated with MUA and Cc molecules. The laser wavelength allowing for the excitation of these substrates is mentioned. C) Resonance TIRSERS spectra of MUA (bottom) and Cc deposited on a MUA SAM (top) for a $532 \mathrm{~nm}$ irradiation. The dashed lines indicate the wavenumber of expected Cc marker bands.

Figure 1C presents resonance TIR-SERS spectra of pristine and Cc-covered MUA SAMs. Hydrophilic MUA SAMs have been chosen to mimic the negatively-charged surface of Cc oxidase, in order to obtain a good recognition of $\mathrm{Cc}$ and facilitate the direct electron transfer process. For this reason, replacing MUA by a mixture of MUA and hydrophobic undecanethiol molecules to better mimic the heterogeneity of real membranes reduces the SERS signal intensity, but the SERS fingerprint itself with specific wavenumbers is not affected (Figure S2 in 
Supporting Information). In the absence of $\mathrm{Cc}$, the successful functionalization with MUA molecules is revealed by the intense band centered at $706 \mathrm{~cm}^{-1}$ assigned to C-S stretching vibrations as well as the band at $1099 \mathrm{~cm}^{-1}$ assigned to the $\mathrm{C}-\mathrm{O}$ stretching vibration of the MUA terminal -COOH/-COO- group. ${ }^{15}$ Carbonyl bands of MUA molecules expected in the 1700-1740 $\mathrm{cm}^{-1}$ were not observed. This may be related to their low Raman cross-section and to the presence of negatively charged terminal carboxylate groups instead their hydrogenated counterparts, thus confirming the relevance of MUA to mimic the surface of Cc oxidase. After deposition of Cc molecules, many new SERS bands centered at 751, 1372, 1584 and $1636 \mathrm{~cm}^{-1}$ and assigned to the porphyrin ring appear. These four marker bands are assigned to the so-called $v_{15}, v_{4}, v_{2}$ and $v_{10}$ vibration modes of the heme group respectively, and are fully consistent with the presence of $\mathrm{Cc}$ in the native ferric $6 \mathrm{cLS}$ state, ${ }^{6,12}$ as expected in the deposited neutral-pH Cc aqueous solution and on SERS-active substrates at $0 \mathrm{~V}$ electrical potential. ${ }^{6}$ Previous Raman measurements reported also a $v_{3}$ band at $1502 \mathrm{~cm}^{-1}$ typical of this state, ${ }^{6}$ but it is hardly discernable in our SERS spectra. It is important to point out that native and non-native forms of Cc with different axial ligands (upon replacement of the labile Met residue by His for instance) and biological activities may both be $6 \mathrm{cLS}$ species. ${ }^{6}$ Thus, only very subtle Raman shifts, requiring a very high $\left(<1 \mathrm{~cm}^{-1}\right)$ spectral resolution to be identified, allow them to be distinguished from each other. However, conventional spectral resolutions are sufficient to exclude indubitably the presence of other species such as $6 \mathrm{cHS}$ ferric, 5cHS ferric, 6cLS ferrous and $5 \mathrm{cHS}$ ferrous states of $\mathrm{Cc}$.

TIR-Raman characterization of $C c$. For the study of $\mathrm{Cc}$, a glass/Au $(10 \mathrm{~nm}) / \mathrm{MUA} / \mathrm{Cc}(5-20 \mathrm{~nm})$ multilayer sample has been selected for three reasons. As explained in the preceding section, $\mathrm{Cc}$ molecules have a good affinity for hydrophilic MUA-coated Au films. Furthermore, in order to 
work in TIR, the critical angle must be precisely determined. However, as the refractive index of $\mathrm{Cc}$ on glass is very close to that of borosilicate glass alone (1.44 vs. 1.518$),{ }^{16,17}$ it turns out to be extremely difficult to identify the glass/Cc interface in the absence of Au interlayer, and thus to identify the TIR regime. In practice, the intensity of the Raman signal associated with a glass/Cc sample was found to be almost constant irrespective of the incidence angle, albeit slightly higher (by $30 \%$ at most) under $p$-polarized than under $s$-polarized light excitation. Finally, the thickness of the Au interlayer was optimized to lead to the strongest Raman signal in TIR configuration. The determination of the ideal thickness not only requires the calculation of mean-square electric fields within the $20 \mathrm{~nm}$-thick Cc layer but also that of the transmittance of the Au interlayer, since the collected Raman signal must traverse the Au film to be detected. Considering these two quantities, the strongest TIR-Raman signal is found for 10-20 nm Au thicknesses under $p$ polarized $561 \mathrm{~nm}$ excitation and $5 \mathrm{~nm}$ Au thickness under $s$-polarized $561 \mathrm{~nm}$ excitation (Figure S3). Note that the $561 \mathrm{~nm}$ wavelength has been preferred to the $532 \mathrm{~nm}$ one for reference TIRRaman characterizations because it allows for the excitation of the LSPR of our Ag/Au TERS probes $^{18}$ and of Cc (Figure 1A), while their excitation would be inefficient at $532 \mathrm{~nm}$. Figure 2A presents mean-square electric fields inside a $20 \mathrm{~nm}$-thick Cc layer covering a $10 \mathrm{~nm}$-thick $\mathrm{Au}$ layer under $p$-polarized and s-polarized light irradiation. Figure 2B shows the corresponding transmittance of the same Au layer. The maximal mean-square electric field is found at $48^{\circ}$ and $41^{\circ}$ incidence angles for $p$-polarized and $s$-polarized excitations, respectively, considering the $41^{\circ}$ critical angle in our configuration. On the experimental standpoint, the dependence of the Raman intensity associated with a glass/Au $(10 \mathrm{~nm}) / \mathrm{MUA} / \mathrm{Cc}$ multilayer sample as a function of the incidence angle can be estimated by integrating the spectral regions centered either at $\sim 1600$ $\mathrm{cm}^{-1}$ (Figures 2C and S4) or at $750 \mathrm{~cm}^{-1}$ (Figures S4 and S5) that are typical of Cc molecules. 
Incidence angles leading to maximal Raman intensities appear to be $46-47^{\circ}$ and $42-43^{\circ}$ under $p$ polarized and s-polarized $561 \mathrm{~nm}$ laser light, which is very close to aforementioned theoretical values. Figure $2 \mathrm{C}$ also shows that the Raman intensity under $p$-polarized excitation is more than twice as high as its counterpart under s-polarized excitation. As p polarization (with dominant component in the z-direction perpendicular to the xy sample plane) is particularly convenient to excite TERS tips in bottom-illumination configuration, ${ }^{19}$ the high mean-square electric field under $p$-polarized excitation constitutes an obvious advantage to probe Cc in TIR-TERS.
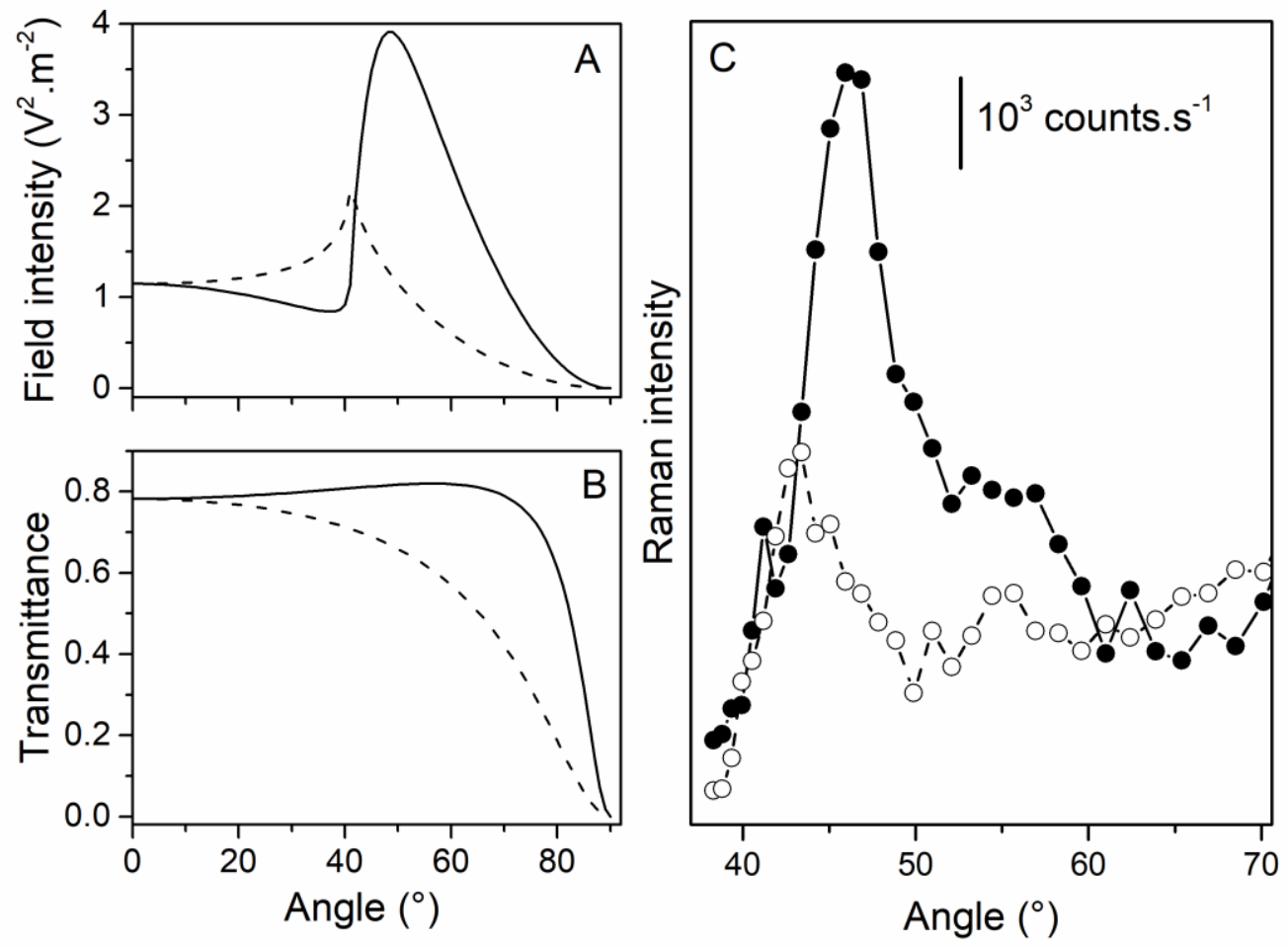

Figure 2. A) Calculated mean-square electric fields in a $20 \mathrm{~nm}$-thick Cc layer on top of a $10 \mathrm{~nm}$ thick $\mathrm{Au}$ film for $p$-polarized (straight line) and $s$-polarized (dashed line) incident light excitations and different incidence angles. B) Calculated transmittance of a $10 \mathrm{~nm}$-thick gold film for $p$-polarized (straight line) and $s$-polarized (dashed line) incident light excitations and 
different incidence angles. C) Raman intensity of the spectral region centered at $1600 \mathrm{~cm}^{-1}$ for a 5-20 nm-thick Cc layer on a $10 \mathrm{~nm}$-thick Au film excited using $p$-polarized (straight line with full circles) and $s$-polarized (dashed line with open circles) $561 \mathrm{~nm}$ incident laser light and different incidence angles.

Performances of TIR-TERS. In order to optimize the intensity of the TERS signal by benefiting especially from any potential gap mode, ${ }^{20}$ a noble metal tip must be placed a few nanometers above the glass/Au/MUA/Cc multilayer sample, where the $\mathrm{Cc}$ coating is limited to a monolayer (or even a submonolayer). The distance between the tip end and the Au layer is then $\sim 6 \mathrm{~nm}$, owing to the $\sim 5 \mathrm{~nm} \mathrm{MUA} / \mathrm{Cc}$ layer and the $1 \mathrm{~nm}$ tip-surface distance. Experimental $\operatorname{Ag}(30 \mathrm{~nm}) / \mathrm{Au}(5 \mathrm{~nm})$ silicon tips are modelled by $\mathrm{Ag}$ tips showing a LSPR at $561 \mathrm{~nm}$, as observed in practice. ${ }^{18}$ FDTD simulations then reveal the occurrence of several phenomena (Figure 3). Under $p$-polarized light excitation in TIR configuration (bottom-right side in Figure 3A), the high intensity of the electromagnetic field on the left side of the tip originates from the surface plasmon enhanced drag effect induced by the incident electric field (Figure $3 \mathrm{~A}$ ). ${ }^{21}$ In addition, a significant electric field enhancement is generated in the $60 \mathrm{~nm}$-broad portion of the MUA/Cc molecular layer below the tip end, which highlights the interest of TIR-TERS (Figure 3A and 3B). The highest value of the average electromagnetic enhancement induced by the Ag tip $\left(|\mathrm{E}| /\left|\mathrm{E}_{0}\right| \sim 5.64\right)$ is indeed obtained for a $42^{\circ}$ incidence angle (Figures $3 \mathrm{C}$ and $3 \mathrm{D}$ ), and corresponds to a total electromagnetic enhancement of $\left(|\mathrm{E}| /\left|\mathrm{E}_{0}\right|\right)^{4} \sim 1.01 \times 10^{3}$. This $42^{\circ}$ angle is very close to the $41^{\circ}$ experimental critical angle. At that $42^{\circ}$ value, a $250 \mathrm{~nm}$-long portion of the Ag tip should be excited by the evanescent field (Figure S6). The resulting lightning-rod effect should thus be increased with respect to the one arising from TIR excitations at wider incidence angles, for which shorter tip portions are excited. This could explain the strong total field enhancement at 
$42^{\circ}$ in TIR configuration. This promising property is highly advantageous for TERS. As the laser power density is $\sim 560$ times lower for TIR irradiation than for classical direct illumination (see SI for deeper explanation), the far-field contribution is strongly reduced in TIR and can even be undetectable, as it is the case for the present glass/Au/MUA/Cc multilayer sample (vide infra). This low fluence avoids any degradation of light sensitive molecules, such as Cc that has to be resonantly excited. Regarding the TERS (near-field) signal however, this fluence is at least partly compensated by the significant enhancement of the electric field allowing for intense enough TERS signatures to be collected (Figure 4). 


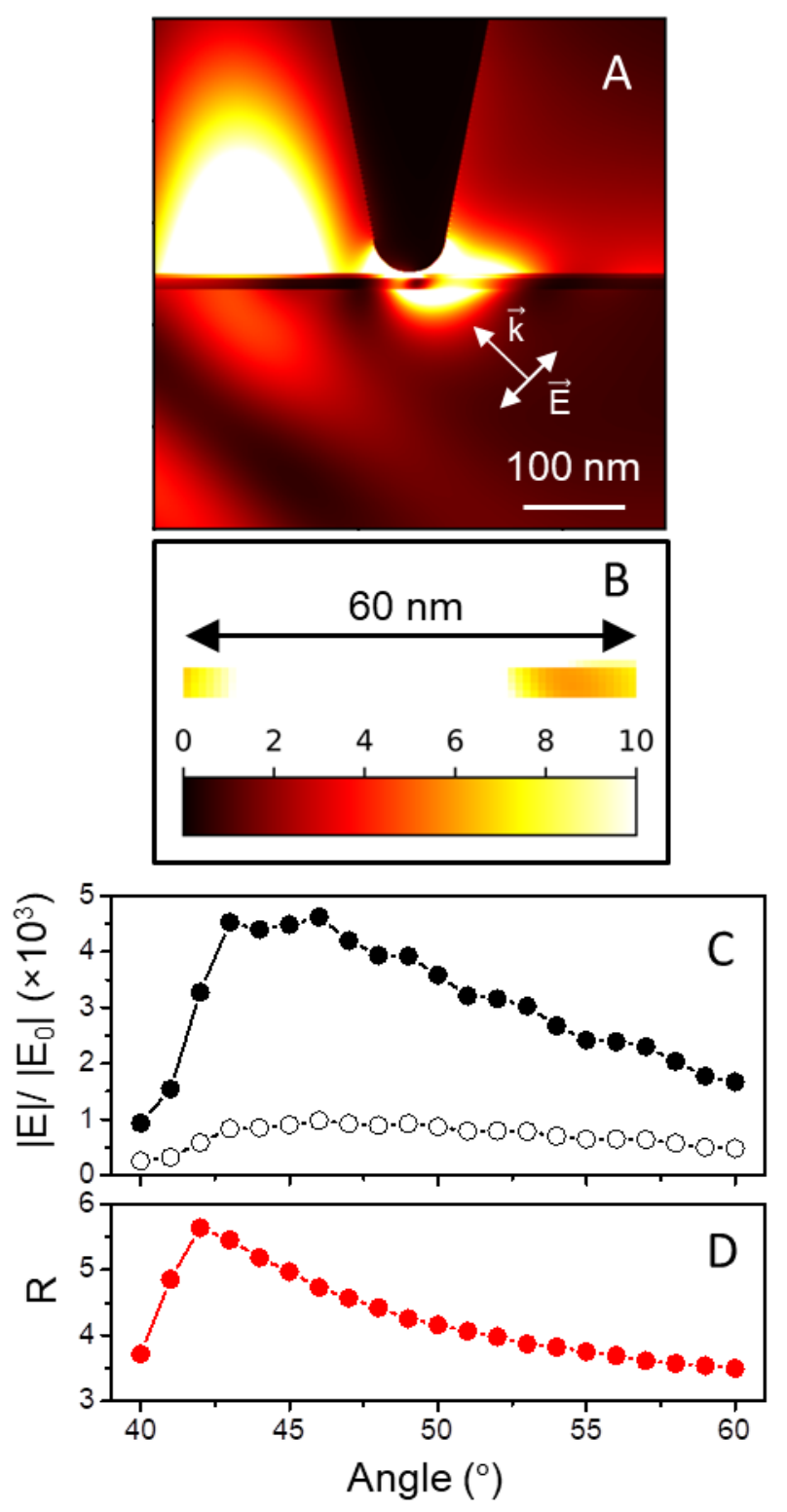

Figure 3. A) FDTD spatial distribution of the electric field magnitude $|\mathrm{E}| /\left|\mathrm{E}_{0}\right|$ when a silver tip is positioned $1 \mathrm{~nm}$ above a $5 \mathrm{~nm}$-thick molecular layer deposited on a $10 \mathrm{~nm}$-thick Au layer, for a $561 \mathrm{~nm}$ light excitation in TIR (with a $46^{\circ}$ incidence angle). Directions of $\overrightarrow{\mathrm{k}}$ and $\overrightarrow{\mathrm{E}}$ associated with the incident $p$-polarized light excitation are indicated. B) Electric field magnitudes in a 60 nm-broad portion of the molecular layer for TIR light excitation. C) Variation of the $|\mathrm{E}| /\left|\mathrm{E}_{0}\right|$ value integrated over the $60 \mathrm{~nm}$-broad region plotted in B as a function of the incidence angle in 
the presence (full circles) and in the absence (open circles) of Ag tip. D) Ratio $R=\left(|E| /\left|E_{0}\right|\right)_{\text {tip }}$ engaged $/\left(|\mathrm{E}| /\left|\mathrm{E}_{0}\right|\right)_{\text {tip withdrawn }}$ as a function of the incidence angle calculated from data plotted in $\mathrm{C}$.

TIR-TERS characterization of Cc. On the experimental standpoint, the maximal TERS signal is found just above the $41^{\circ}$ critical angle $\left(\sim 42^{\circ}\right)$, in excellent agreement with FDTD calculations. Figure 4A presents five TIR-TERS spectra obtained with different TERS probes. The first striking difference from one spectrum to another relates to relative intensities of TERS bands. This is a common behavior in TERS due in particular to the nanoscale confinement of the electromagnetic field at the tip apex and the imperfect homogeneity of the sample. ${ }^{19}$ Indeed, the lateral spatial resolution is better than $10 \mathrm{~nm}$ using our metallized TERS probes, irrespective of the curvature radius of the TERS tip apex. ${ }^{18,19}$ This relatively constant value suggests that the size of TERS-active nanoparticles at the tip apex remains similar. However, depending on the nanoparticle shape, different TERS signatures may appear owing to the sensitivity of the TERS signal to the polarization of the excitation light and the orientation of molecular vibration modes. Furthermore, the spatial resolution is larger than the size of a single $\mathrm{Cc}$ molecule and the $\mathrm{Cc}$ coverage on the MUA SAM is slightly submonolayer. Thus, the contribution of MUA and Cc molecules in TERS spectra may differ depending of the position of the TERS tip on the sample. The resulting intensity fluctuations are also consistent with the distinct relative intensities observed in TERS spectra reported in the literature for Cc. ${ }^{12-14}$ Despite this drawback, the $v_{15}, v_{4}$, $v_{3}, v_{2}$ and $v_{10}$ vibration modes can be discerned in most TERS spectra (Figure 4B, Tables S1 and $\mathrm{S} 2$ in SI). TERS wavenumbers of these Cc marker bands are very close to their SERS counterparts $\left(751 \pm 4 \mathrm{~cm}^{-1}, 1375 \pm 3 \mathrm{~cm}^{-1}, 1497 \pm 4 \mathrm{~cm}^{-1}, 1588 \pm 3 \mathrm{~cm}^{-1}\right.$ and $1632 \pm 2 \mathrm{~cm}^{-1}$ instead of $751 \mathrm{~cm}^{-1}, 1372 \mathrm{~cm}^{-1}, 1505 \mathrm{~cm}^{-1}, 1584 \mathrm{~cm}^{-1}$ and $1636 \mathrm{~cm}^{-1}$ for $v_{15}, v_{4}, v_{3}, v_{2}$ and $v_{10}$ vibration modes, respectively). Other SERS vibrations modes of Cc, such as those centered at $1024 \pm 3 \mathrm{~cm}^{-}$ 
${ }^{1}, 1180 \pm 7 \mathrm{~cm}^{-1}, 1238 \pm 3 \mathrm{~cm}^{-1}$ and $1404 \pm 4 \mathrm{~cm}^{-1}$ assigned to $v_{31}, v_{30}, v_{13}$ and $v_{20}$ heme vibration modes respectively, also emerge in several TERS spectra. A few TERS bands below $700 \mathrm{~cm}^{-1}$, at $\sim 861 \mathrm{~cm}^{-1}, \sim 1356 \mathrm{~cm}^{-1}$ and $\sim 1482 \mathrm{~cm}^{-1}$ are not discerned in Raman and SERS spectra. They are ascribed to amino acid residues, and especially to Tyr vibrations modes (Tables S1 and S2), that can be efficiently probed in TERS. ${ }^{1,12}$ All the other TERS bands can be attributed to vibrations of MUA molecules (Tables S1 and S2). Figure 4 thus demonstrates that SERS and TERS spectra of $\mathrm{Cc}$ in its $6 \mathrm{cLS}$ state are consistent to each other, and confirm the often admitted law that SERS vibration modes can be considered as good references to assign TERS bands of biomolecules. Finally, this study also reveals the possibility to specifically identify the 6cLS ferric state of Cc from other states with the nanoscale spatial resolution of TERS and the low-fluence excitation in TIR, which is of high interest for future investigations on biological tissues.
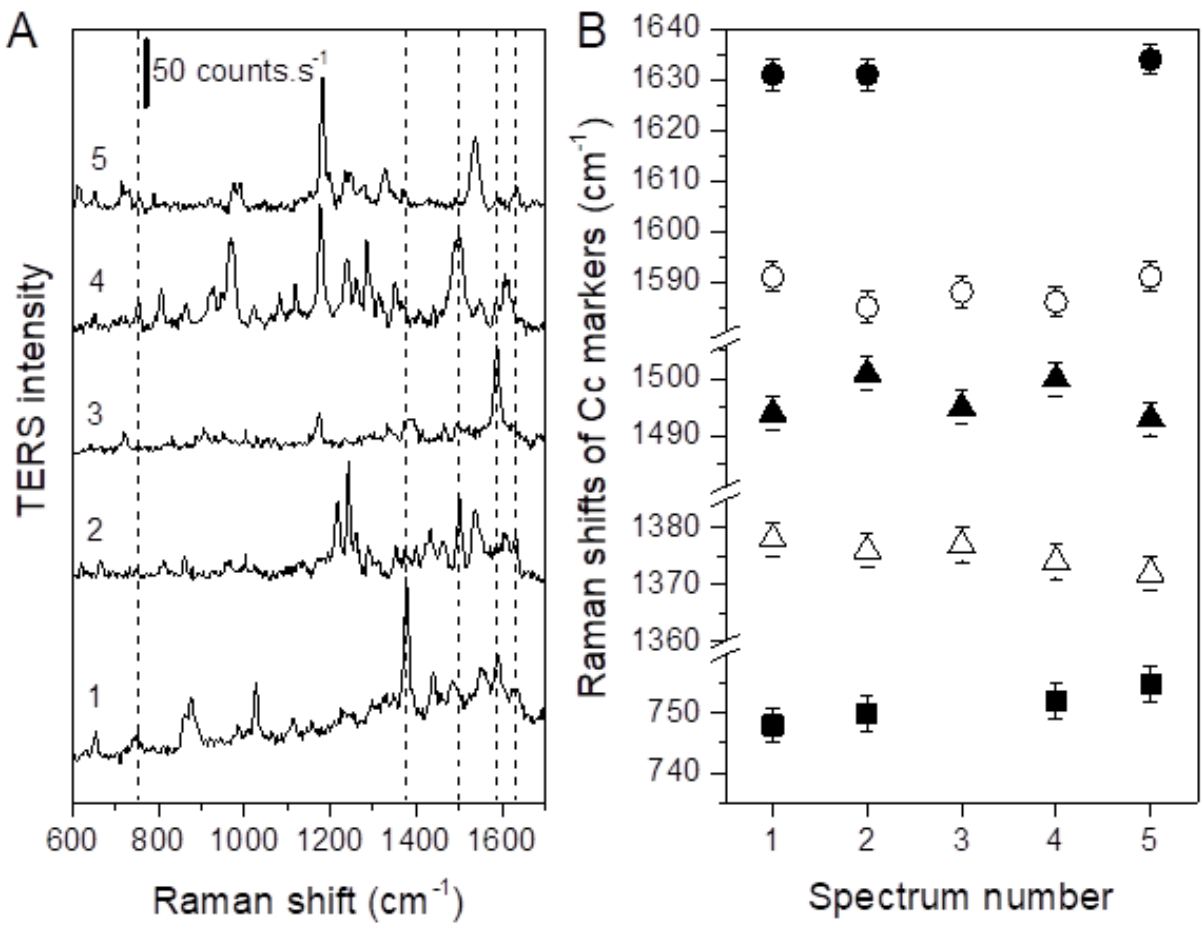
Figure 4. A) TIR-TERS spectra of a glass/Au/MUA/Cc sample obtained by using different metal-coated tapping-mode AFM tips, for an incidence angle just above the critical angle. Dashed lines are guides for the eye that indicates the position of $v_{15}, v_{4}, v_{3}, v_{2}$ and $v_{10}$ vibration modes. B) Variation of the Raman shifts associated with $v_{15}$ (full squares), $v_{4}$ (open triangles), $v_{3}$ (full triangles), $v_{2}$ (open circles) and $v_{10}$ (full circles) vibration modes for the five spectra plotted in $\mathrm{A}$.

In summary, we have demonstrated that performing TERS in TIR configuration is an efficient way to probe biomolecules with minimal incident laser power densities to avoid any sample degradation. In the case of $\mathrm{Cc}$, the native $6 \mathrm{cLS}$ form could be nicely characterized and its spectral signature appeared to closely match that found in TIR-SERS experiments, performed for the first time on a biomolecule as well. Our results thus suggest that 6cLS species of Cc, potentially implicated in the apoptotic processes, could be detected on oxidized lipid membranes with nanoscale spatial resolution. Finally, as TIR-SERS has been recently proven to be a particularly promising technique for investigations in liquid medium, ${ }^{22}$ the next challenge would be to develop the TIR-TERS technology for studies in this biologically relevant environment.

\section{ASSOCIATED CONTENT}

\section{Supporting Information.}

The following files are available free of charge.

Experimental and theoretical details, Interactions between Cc and thiol SAMs linked to electron transfer, resonance SERS spectra of Cc deposited on mixed MUA/undecanethiol (UT) SAMs, Theoretical calculations of mean-square electric fields in a Cc layer and transmittances in $\mathrm{Au}$ 
layers, Conventional Raman measurements in TIR geometry under $p$-polarized and $s$-polarized light excitations, Calculation of the power density in TIR and classical Raman configurations, Near-field nature of the excitation of the TERS probe showing the spatial extent of the evanescent field as a function of the incidence angle, Comparison of TIR-Raman, TIR-SERS and TIR-TERS vibration modes. (PDF)

\section{AUTHOR INFORMATION}

\section{Corresponding author}

*Email: sebastien.bonhommeau@u-bordeaux.fr

\section{ORCID}

Sébastien Bonhommeau: 0000-0002-9213-7201

Sophie Lecomte 0000-0001-8310-4849

\section{Notes}

The authors declare no competing financial interests.

\section{ACKNOWLEDGMENT}

This study has been carried out with financial support from the French State, managed by the French National Research Agency (ANR) in the frame of "the Investments for the future" Programme IdEx Bordeaux - ANR-10-IDEX-03-02. All the Raman, SERS and TERS measurements were performed at the platform SIV (Spectroscopie et Imagerie Vibrationnelle) at the University of Bordeaux, funded by the European Union (FEDER) and the Nouvelle Aquitaine region.

\section{REFERENCES}


(1) Bonhommeau, S.; Lecomte, S. Tip-Enhanced Raman Spectroscopy: a Tool for Nanoscale Chemical and Structural Characterization of Biomolecules. ChemPhysChem 2018, 19, 818.

(2) Heilman , A.L.; Gordon, M.J. Tip-Enhanced Near-Field Optical Microscope with Side-on and ATR-mode sample excitation for super-resolution Raman imaging of surfaces. $J$. Appl. Phys. 2016, 119, 223113.

(3) Fedurco, M. Redox Reactions of Heme-Containing Metalloproteins: Dynamic Effects of Self-Assembled Monolayers on Thermodynamics and Kinetics of Cytochrome C Electron-Transfer reactions. Coord. Chem. Rev. 2000, 209, 263-331.

(4) Green, D.R. Apoptotic Pathways: the Roads to Ruin. Cell 1998, 94, 695-698.

(5) Hengartner, M.O. The Biochemistry of Apoptosis. Nature 2000, 407, 770-776.

(6) Oellerich, S.; Wackerbarth, H.; Hildebrandt, P. Spectroscopic Characterization of Nonnative Conformational States of Cytochrome C. J. Phys. Chem. B 2002, 106, 65666580 .

(7) Murgida, D.H.; Hildebrandt, P. Proton-Coupled Electron Transfer of Cytochrome C. J. Am. Chem. Soc. 2001, 123, 4062-4068.

(8) Murgida, D.H.; Hildebrandt, P.; Wei, J.; He, Y.-F.; Liu, H.; Waldeck, D.H. SurfaceEnhanced Resonance Raman Spectroscopic and Electrochemical Study of Cytochrome C Bound on Electrodes through Coordination with Pyridinyl-Terminated Self-Assembled Monolayers. J. Phys. Chem. B 2004, 108, 2261-2269.

(9) Lecomte, S.; Hildebrandt, P., Soulimane, T. Dynamics of the Heterogeneous ElectronTransfer reaction of Cytochrome C552 from Thermus thermophilus. A Time-Resolved Surface-Enhanced resonance Raman Spectroscopic Study. J. Phys. Chem. B 1999, 103, 10053-10064. 
Hobara, D.; Niki, K.; Zhou, C.; Chumanov, G.; Cotton, T.M. Characterization of Cytochrome C Immobilized on Modified Silver and Gold Electrodes by SurfaceEnhanced Raman Spectroscopy. Colloid Surf A 1994, 93, 241-250. Xu, L.-J.; Zong, C.; Zheng, X.-S.; Hu, P.; Feng, J.M.; Ren, B. Label Free Detection of Native Proteins by Surface-Enhanced Raman Spectroscopy Using IodideModified Nanoparticles. Anal. Chem. 2018, 86, 2238-2245. Yeo, B.-S.; Mädler, S.; Schmid, T.; Zhang, W.; Zenobi, R. Tip-Enhanced Raman Spectroscopy Can See More: The Case of Cytochrome C. J. Phys. Chem. C 2008, 112, 4867-4873.

(13) Tanabe, I.; Egashira, M.; Suzuki, T.; Goto, T.; Ozaki, Y. Prevention of Photooxidation of Deoxymyoglobin and Reduced Cytochrome C during Enhanced Raman Measurements: SERRS with Thiol-Protected Ag nanoparticles and a TERS Technique. J. Phys. Chem. C 2014, 118, 10329-10334.

Böhme, R.; Mkandawire, M.; Krause-Buchholz, U.; Rösch, P.; Rödel, G.; Popp, J.; Deckert, V. Characterizing Cytochrome C States - TERS Studies of Whole Mitochondria. Chem. Commun. 2011, 47, 11453-11455. Socrates, G. Infrared and Raman Characteristic Group Frequencies: Tables and Charts; John Wiley \& Sons Ltd.: Chichester, 2001.

Runge, A.F.; Rasmussen, N.C.; Saavedra, S.S.; Mendes, S.B. Determination of Anisotropic Optical Constants and Surface Coverage of Molecular Films Using Polarized Visible ATR Spectroscopy. Application to Adsorbed Cytochrome c Films. J. Phys. Chem. B 2005, 109, 424-431. Dadafarin, H.; Konkov E.; Omanovic, S. Electrochemical Functionalization of a 316L Stainless Steel Surface with a 11-mercaptoundecanoic Acid Monolayer: Stability Studies. Int. J. Electrochem. Sci. 2013, 8, 369-389. 
Najjar, S.; Talaga, D.; Schué, L.; Coffinier, Y.; Szunerits, S.; Boukherroub, R.;

Servant, L.; Rodriguez, V.; Bonhommeau, S. Tip-Enhanced Raman Spectroscopy of Combed Double-Stranded DNA Bundles. J. Phys. Chem. C 2014, 118, 1174-1181.

Talaga, D.; Smeralda, W.; Lescos, L.; Hunel, J.; Lepejova-Caudy, N.; Cullin, C.;

Bonhommeau, S.; Lecomte, S. PIP 2 Phospholipid-Induced Aggregation of Tau Filaments Probed by Tip-Enhanced Raman Spectroscopy. Angew. Chem. Int. Ed. 2018, 57, 1573815742.

Stadler, J.; Oswald, B.; Schmid, T.; Zenobi, R. Characterizing Unusual Metal Substrates for Gap-Mode Tip-Enhanced Raman Spectroscopy. J. Raman Spectrosc. 2013, 44, 227-233.

Vengurlekar, A.S.; Ishihara, T. Surface plasmon enhanced photon drag in metal films. Appl. Phys. Lett. 2005, 87, 091118. Plissonneau, M.; Madeira, A.; Talaga, D.; Bonhommeau, S.; Servant, L.; Vallée, R.A.L.; Labrugère, C.; Goldthorpe, I.A.; Pautrot-D’Alencon, L.; Lemercier, T.; TreguerDelapierre, M. Efficient Passivation of Ag Nanowires with 11-Mercaptoundecanoic Acid Probed Using In Situ Total-Internal-Reflection Surface-Enhanced Raman Scattering Spectroscopy. ChemNanoMat 2019, 5, 1044-1049. 